\title{
ARTÍGULO ORIGINAL: PREVALENGIA, FACTORES DE RIESGO Y MORTALIDAD DE LOS PAGIENTES GON GANDIDEMIA ATENDIDOS EN EL HOSPITAL SAN JUAN DE DIOS DURANTE ENERO 2008 A DICIEMBRE 2010
}

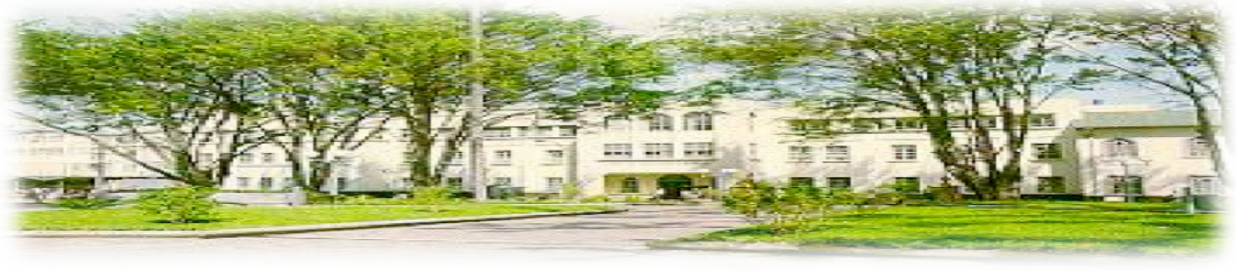

Hospital San quan de Dias. San José. Costa Rica. Fundado en 1845

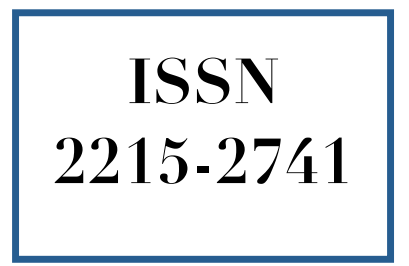

Recibido: Aceptado:
$30 / 06 / 2012$

$18 / 07 / 2012$

\author{
Vanessa Zárate Elizondo 1 \\ Manuel Antonio Villalobos Zúñiga ${ }^{2}$ \\ Nury Mora Brenes ${ }^{3}$
}

\footnotetext{
${ }^{\mathbf{1}}$ Especialista en Medicina Interna. Residente de Infectología UCR-CENDEISSS

${ }^{2}$ Especialista en Medicina Interna e Infectología, Hospital San Juan de Dios. San José Costa Rica. Correo electrónico: manue1701@gmail.com. Correo: 2434-1000 San José.

${ }^{3}$ Microbióloga Química Clínica, especialiste en Micología. Laboratorio Clínico. Hospital San Juan de Dios
}

\section{RESUMEN}

La Candidemia, constituye una de las infecciones nosocomiales del torrente sanguíneo más importantes en los pacientes hospitalizados, asociados a una alta morbimortalidad. En el Hospital San Juan de Dios (HSJD) los últimos datos registrados datan de 1999, se realizó un estudio retrospectivo, de tipo descriptivo. Se basó en la revisión de expedientes clínicos de los pacientes documentados con un hemocultivo positivo por Candida $s p$, ingresados en este nosocomio durante un periodo de 3 años, entre Enero 2008 a Diciembre 2010.

Se logró determinar las especies de Candida más frecuentes, los factores de riesgo principales, la mortalidad atribuible a dicha causa y el tratamiento administrado en cada caso.

\section{PALABRAS CLAVE}

Candida. Candidemia. Prevalencia. Catéter venoso central.

\section{ABSTRACT}

Candidemia is one of most important systemic infections in hospitalized patients and it is associated with a high risk of mortality. In San Juan de Dios Hospital, the last data collected in this matter, last from 1999. Because of this, a retrospective and descriptive study was made. It was based on medical files information, from patients with a positive blood culture, where Candida sp. was identified. The period of study was between January 2008 and December 2010, a 3 years period. 
The most frequent species of Candida, the main risk factors for this kind of infection, the mortality associated with this disease and the treatment administered to the patients were the most important issues assessed.

\section{KEY WORDS}

Candida. Candidemia. Prevalence. Central venous catheter.

\section{INTRODUCCIÓN}

La Candidemia, se define como la infección sanguínea causada por diversas especies del género Candida sp; es una infección de salud seria y se asocia con un peor pronóstico ${ }^{(2)}$. La incidencia y por ende la prevalencia de la candidemia se ha incrementado de forma significativa en los últimos años, en los EUA y Europa representa la cuarta causa de infección sanguínea nosocomial $^{(3)}$; lo anterior dado principalmente por el uso de métodos diagnósticos y terapéuticos invasivos, por el empleo de tratamientos agresivos con antibióticos de amplio espectro y agentes quimioterapéuticos, por el desarrollo de unidades de cuidado intensivo, así como por el aumento en la sobrevida de los pacientes con enfermedades crónicas ${ }^{(1,3,7,8)}$.

La mayoría de estas infecciones son severas, rápidamente progresivas, difíciles de diagnosticar y de tratar.

Así las infecciones del género Candida se han incrementado sustancialmente en las últimas dos décadas; constituye una causa importante de morbimortalidad entre los pacientes severamente enfermos, causando además un aumento en la estancia hospitalaria con la consecuente elevación de los costos hospitalarios ${ }^{(3,4,9-11,15)}$.

Candida albicans se mantiene como la causa más frecuente de fungemias, pero se ha observado un aumento de aquellas causadas por Candidas no albicans ${ }^{(1,3,4,11,15,20)}$.

La frecuencia reportada por especies es: C. albicans 40-60\%, C. glabrata 20-30\%, C. krusei 5$10 \%$, C. lusitaniae $0-5 \%, C$. parapsilosis 10 $20 \%$ y C. tropicalis $20-30 \%{ }^{(9)}$
Los principales factores de riesgo para la candidemia incluyen la exposición previa a antibióticos de amplio espectro, la presencia de catéter venoso central, el uso de nutrición parenteral total, exposición a quimioterapia, el ingreso a la Unidad de Cuidados intensivos, Cirugía previa sobre todo de tipo abdominal, transfusiones sanguíneas repetidas, entre otros ${ }^{(1-4,15)}$.

El diagnóstico para la candidemia requiere de la sospecha diagnóstica, la presencia de los factores de riesgo y la evidencia microbiológica de dicho germen en el hemocultivo ${ }^{(1,2,7,8,15)}$.

Antes del advenimiento de la era del tratamiento antifúngico, la mortalidad por candidemia era prácticamente del $80 \%$, hoy en día, se ha calculado que la mortalidad atribuible a la candidiasis invasiva representa un porcentaje tan alto como un 47\%; aunque muchas autoridades calculan que la mortalidad atribuible es de $15 \%$ a $25 \%$ para adultos $^{(14)}$.

Debido a que no existen estudios recientes en el Hospital San Juan de Dios sobre este problema, este trabajo fue realizado inicialmente con el objetivo de determinar la prevalencia, factores de riesgo y mortalidad de los pacientes con candidemia atendidos en el Hospital San Juan de Dios durante Enero 2008 a Diciembre 2010.

\section{DISEÑO DEL ESTUDIO}

La investigación fue de carácter retrospectivo y descriptivo, basada en la revisión de expedientes clínicos y análisis de los mismos, mediante una hoja de recolección de datos previamente estructurada. La selección de los expedientes se realizó mediante el listado de los reportes de los hemocultivos positivos de los pacientes facilitada por el laboratorio clínico, división de Micología del Hospital San Juan de Dios y posteriormente fueron facilitados por el archivo del hospital, lo anterior en el periodo estipulado para el estudio.

\section{Criterios de Inclusión:}

Se incluyó todos los pacientes mayores de 12 años que tenían documentado en el expediente un hemocultivo positivo por Candida sp; y que fueron atendidos en el HSJD durante el periodo del estudio, no hubo restricción por género o etnia. 
Criterios de exclusión:

Se excluyó pacientes menores de 12 años de edad, también aquellos en quienes al momento de revisar el expediente clínico, no tenían documentado el hemocultivo positivo por Candida $s p$.

Una vez recolectados los datos, fueron tabulados en una base de datos archivada digitalmente en el paquete EXCEL 12.3, con la definición de las variables planteadas y con una codificación interna para identificar cada sujeto en las hojas de recolección. Con dicho paquete se realizó el análisis descriptivo de los resultados.

Dado que se trata de un estudio retrospectivo, observacional y descriptivo, los métodos estadísticos utilizados se basaron en Estadística Descriptiva.

\section{RESULTADOS}

Se analizaron 96 pacientes, de los cuales 61 cumplieron con los criterios para ser incluidos en el estudio, 4 se excluyeron ya que correspondían a recién nacidos, 16 no tenían el hemocultivo positivo documentado en el expediente y 15 fueron reportados como extraviados en el archivo del hospital.

La presencia de candidiasis sistémica fue de 1,91 por cada 10.000 egresos/año en el Hospital San
Juan de Dios. La mayoría de los casos se presentó en hombres $(32 / 61,52 \%)$ los datos de frecuencia relacionados con la edad se analizan en la figura 1. La mortalidad general fue de $59 \%$ (37/61), siendo principalmente en hombres mayores de 60 años (13/61). No hubo una diferencia significativa entre el número de casos por servicio. En la unidad de cuidados intensivos se fueron diagnosticados 5 casos durante el periodo de estudio.

La fiebre, fue identificada como el síntoma más frecuente $(58 / 61,95 \%)$, seguido por la taquicardia sinusal, definida como una frecuencia cardiaca mayor de 100 latidos por minuto (53/61, 86\%).

La mortalidad atribuible a la candidemia 7 días posterior al diagnóstico fue de $21 \%$ (13/61). La mortalidad atribuible a la candidemia 30 días posterior al diagnóstico fue del 45\% (28/61).

La estancia estuvo en un rango de 15 a más de 40 días, con un promedio de 18 días.

El promedio de días posterior al ingreso, luego de los cuales se presenta un episodio de Candidemia fue de 17 días.

Dentro de las comorbilidades que cuentan como factor de riesgo para el desarrollo de candidemia, la diabetes mellitus tipo 2 fue la más frecuente $(13 / 61,21 \%)$.

Figura 1. Pacientes con candidemia atendidos en el HSJD de enero 2008 a diciembre 2010, por edad y sexo.

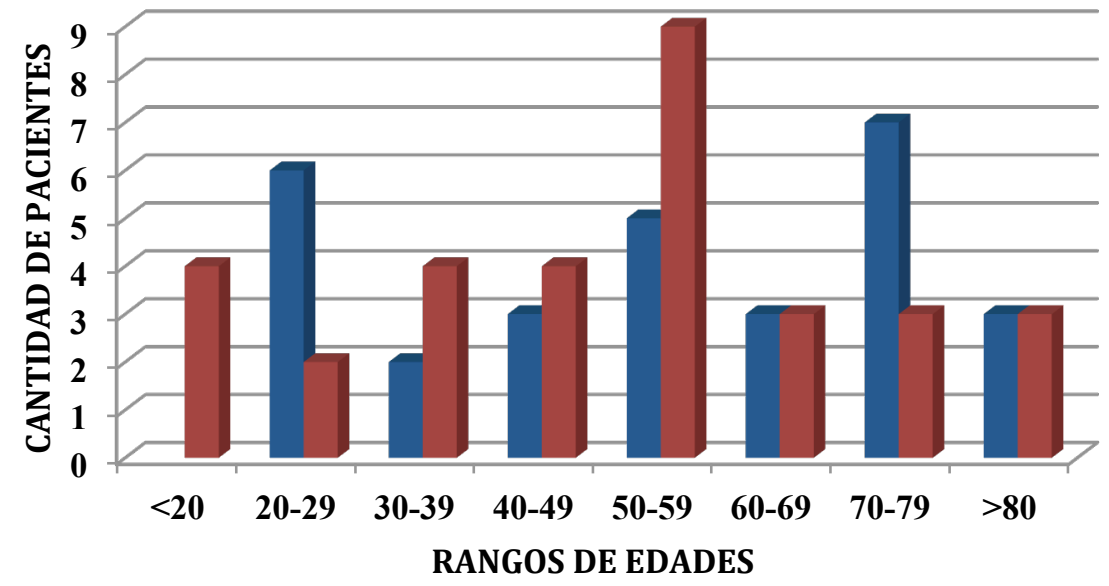

- HOMBRES

- MUJERES 
La colonización previa por Candida sp. no fue consignada en la mayoría de los pacientes durante su estancia hospitalaria, únicamente se documentó algún sitio con presencia de Candida sp en el $11 \%(7 / 61)$.

Dentro de los factores de riesgo encontrados, el uso previo de antibióticos fue el principal (60/61, $98 \%)$, principalmente vancomicina $(50 / 61)$ y con un promedio de administración de 11 días; seguida por el uso de metronidazol endovenoso $(38 / 61)$, gentamicina y otros aminoglicósidos $(28 / 61)$ y cefalosporinas de tercera generación (27/61).

Otros factores de riesgo identificados fueron: presencia de catéter venoso central $(57 / 61,98 \%)$, ventilación mecánica asistida (36/61, 59\%), neoplasias gastrointestinales $(11 / 61,18 \%)$, neoplasias hematológicas con neutropenia $(8 / 61$, $18 \%)$, diabetes mellitus tipo $2(13 / 61,21 \%)$ y nutrición parenteral con un promedio de 30 días de uso $(31 / 61,51 \%)$.

Cuadro 1. Resumen de factores de riesgo para Candidiasis sistémica.

\begin{tabular}{ccc}
\hline Factor de Riesgo & N & $\%$ \\
\hline Uso de antibióticos & 60 & 98 \\
\hline CVC & 57 & 93 \\
VMA & 36 & 59 \\
DM tipo 2 & 13 & 21 \\
\hline NEO Gastrointestinal & 11 & 18 \\
\hline NEO Hematológica & 8 & 13 \\
\hline
\end{tabular}

CVC: catéter venoso central. VMA: ventilación mecánica asistida. NEO: neoplasia

Entre los pacientes con alguna neoplasia hematológica incluidos en el presente estudio (8/61), el recuento promedio de neutrófilos en todos los casos fue menor de $500 / \mathrm{mm}^{3}$, además todos tenían profilaxis anti fúngica con fluconazol oral.

El número promedio de días catéter luego de los cuales la presencia del mismo se asocia con un mayor riesgo para desarrollar candidemia fue de 17.2 días; el sitio anatómico de colocación más frecuentemente usado fue subclavio (54/61) (figura 2).
La cirugía abdominal tipo laparotomía es la que más se asocia con la presencia de candidemia $18 \%(11 / 61)$, en la mayoría de los casos la candidemia se desarrolló en promedio 24 días posterior al procedimiento quirúrgico. Las especies de Candida más frecuentemente aisladas y su relación con factores de riesgo se muestran en el cuadro 2 .

Cuadro 2. Especies de Candida aisladas y su relación con factores de riesgo.

\begin{tabular}{|c|c|c|c|}
\hline Especie & $\mathbf{N}$ & $\%$ & FR principal \\
\hline C. albicans & 22 & 36 & \\
\hline C. parapsilosis & 13 & 21 & $\mathrm{CVC}$ \\
\hline C. tropicalis & 10 & 16 & \\
\hline C. glabrata & 6 & 10 & $\begin{array}{c}\text { Neoplasia } \\
\text { Hematológica }\end{array}$ \\
\hline C. famata & 4 & 7 & \\
\hline C. krusei & 3 & 5 & $\begin{array}{c}\text { Neoplasia } \\
\text { Hematológica }\end{array}$ \\
\hline C. haemulonii & 2 & 3 & \\
\hline C. lusitaniae & 1 & 2 & \\
\hline Total & 61 & 100 & \\
\hline
\end{tabular}

FR: factor de riesgo. CVC: Catéter venoso central

En el presente estudio, el tratamiento empírico, se inicio en el 55,5\% (34/61), en promedio a las $72 \mathrm{hrs}$ posterior al resultado del hemocultivo.

El tratamiento antifúngico de primera línea que más se utilizó fue la Anfotericina B, 49\% (30/61), con un promedio de 2 semanas de duración para una dosis acumulada promedio de $400 \mathrm{mg}$, seguida por la caspofungina intravenosa utilizada durante 10 días en promedio, 21\% (13/61).

El efecto secundario más frecuentemente asociado al uso de Anfotericina B fue la insuficiencia renal aguda $(15 / 61,24.6 \%)$.

Cabe destacar que a ninguno de los casos incluidos en el presente estudio, se le realizó pruebas de sensibilidad antifúngica, tampoco hemocultivos control luego de iniciado el tratamiento antifúngico, como lo establecen las guías para el tratamiento de la candidemia. 
Figura 2. Colocación de catéter como factor de riesgo según sitio anatómico de colocación y duración en días, en pacientes con candidemia, atendidos en el HSJD. Enero 2008-diciembre 2010.

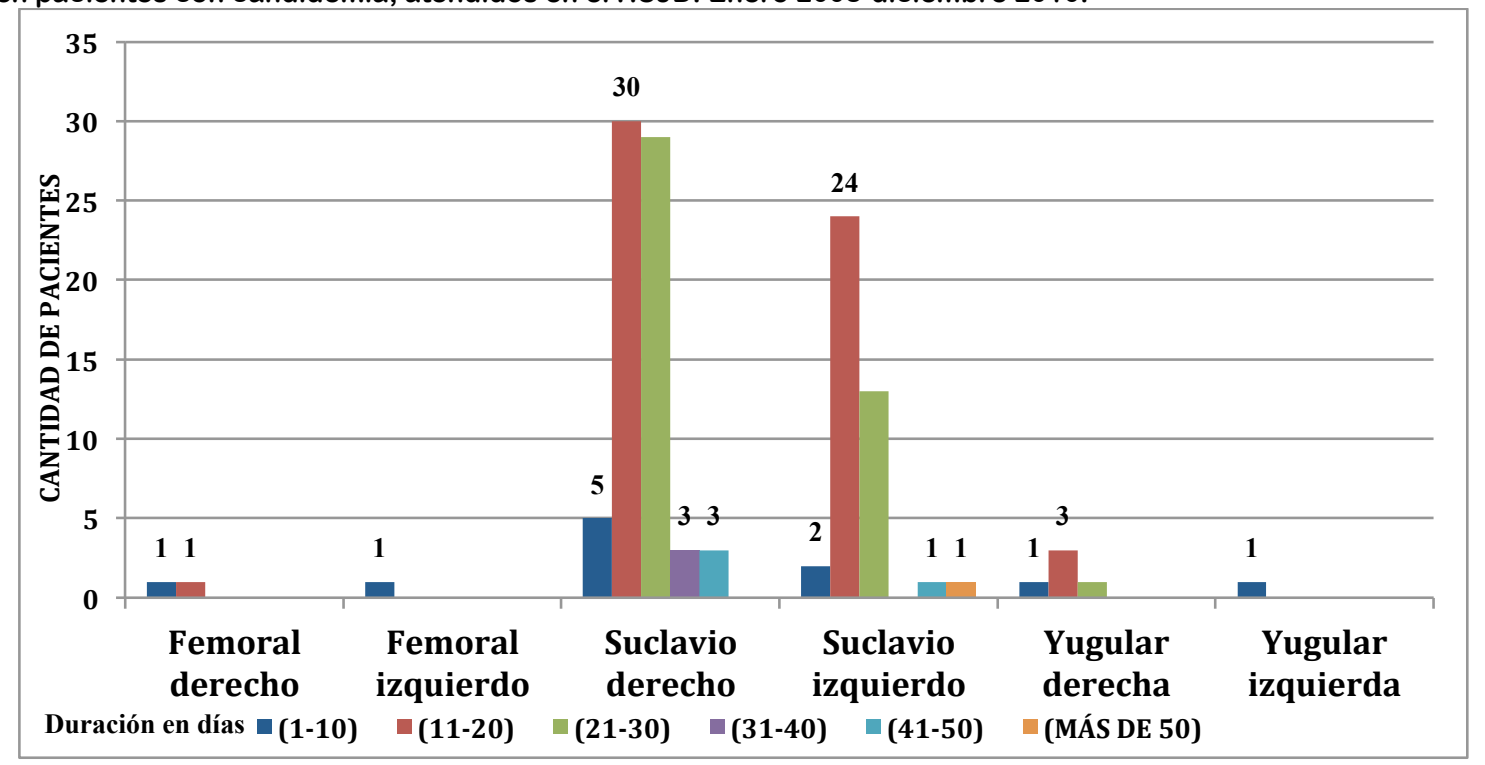

Fuente: Base de datos del proyecto de investigación: Prevalencia, factores de riesgo y mortalidad de los pacientes con candidemia atendidos en el HSJD de enero 2008 a diciembre 2010

\section{DISCUSIÓN}

Durante muchos años, la candidemia fue comúnmente considerada como un evento inusual que ocurría generalmente entre pacientes inmunocomprometidos y con enfermedades de fondo muy severas ${ }^{(1-4,7,10,12)}$. Actualmente esta infección ha cobrado importancia entre pacientes inmunocompetentes y con enfermedades de base no tan severas $^{(2)}$.

Actualmente la candidemia constituye una de las causas más frecuentes de infecciones nosocomiales del torrente sanguíneo, representando el cuarto microorganismo más frecuente causante de hemocultivos positivos en Estados Unidos y el quinto en España ${ }^{(3)}$.

La frecuencia con la que se identificó la candidemia 1,91 por cada 10.000 egresos/año no es despreciable. Tomando en cuenta que en España por ejemplo, según el estudio de Almirante et $a l^{(1)}$, se reporta una prevalencia de 4,3 casos por cada 10.000 egresos/año, además es similar a la reportada en países del norte de Europa 1,7-4,9 casos/10.000 egresos/año ${ }^{(5)}$.

Al igual que en la literatura, pudimos observar un predominio en el sexo masculino y mayores de 65 años $^{(1)}$, los cuales representaron más de la mitad de los casos, seguidos por hombres jóve- nes menores de 30 años, lo cual difiere de datos publicados en la literatura internacional, sin embargo, cabe destacar que los pacientes incluidos en este grupo etario para el presente estudio en su mayoría tenían comorbilidades y factores de riesgo tales como politrauma, sepsis severa, uso prolongado de antibióticos de amplio espectro, nutrición parenteral, ventilación mecánica asistida prolongada o cirugía reciente, los cuales predisponen a la infección, independientemente de la $\operatorname{edad}^{(4)}$.

La mortalidad general es alta, en nuestro estudio fue del $59 \%$, lo anterior es similar a la reportada en la literatura internacional la cual oscila entre un $40 \%$ y $60 \%$ en diferentes trabajos ${ }^{(15)}$, lo que pone de manifiesto que en los últimos años se ha producido un aumento en la incidencia de forma paralela al aumento de los pacientes de riesgo, sin embargo, en dicha mortalidad está incluida la asociada con las enfermedades de base graves que presentan muchos de los pacientes infectados al momento del diagnóstico ${ }^{(11)}$.

La mortalidad de las candidemias, no se ha reducido a pesar de los avances alcanzados en los últimos años y la introducción de nuevos antifùngicos ${ }^{(15)}$, en nuestro estudio la mortalidad atribuible a la candidemia a los 7 días fue del $21 \%$ y a los 30 días del $45 \%$, lo cual es similar a 
lo reportado por otros autores, la cual oscila entre un $7 \%$ y $71 \%$ aproximadamente ${ }^{(4,12)}$.

Según los datos obtenidos en nuestro hospital, en promedio, luego de dos semanas posterior al ingreso es cuando se presenta la mayoría de los casos de candidemia, lo cual coincide con los datos reportados en otras latitudes, cuyo promedio oscila entre los 12 y 14 días, llegando hasta los 22 a 28 dìas en los casos más tardíos. ${ }^{(1,15)}$

Sin duda alguna, el desarrollo de un episodio de candidemia, condiciona estancias hospitalarias prolongadas y un aumento en los costos por estancia hospitalaria, en el presente estudio no fue menor de dos semanas en ninguno de los casos, con un promedio de 18 días, lo anterior ya ha sido descrito en otros países. En general se describe superior a 10 días, pudiendo alcanzar hasta los 30 días de exceso en la hospitalización; por ejemplo en los EUA se ha estimado que el incremento medio del coste por paciente con candidemia sería de alrededor de $\$ 45.000$ y que el gasto anual en dicho país superaría la cifra de 1.7 billones de dólares anuales. ${ }^{(1)}$ Nuestro medio no escapa a dicha realidad, lo cual es un elemento a considerar si se toma en cuenta que en promedio un día de estancia hospitalaria en salón general supera los $\$ 500.000$ diarios (cerca de $\$ 1.000)$.

Desde el punto de vista clínico, la candidemia se comporta en forma similar a las septicemias bacterianas, hecho que se demuestra por el gran porcentaje de pacientes que se presentó con fiebre y taquicardia inducida por sepsis. Los episodios de candidemia que se presentaron sin estas manifestaciones no deben desestimarse, ya que la retinitis por ejemplo, tambíen puede presentarse. Sin embargo, las guías de tratamiento establecen que todos los pacientes con candidemia deben someterse a una evaluación oftalmológica con dilatación de pupila, para descartar una endoftalmitis por Candida. Sin embargo en el presente estudio, a ninguno de los pacientes se les realizó dicha valoración por oftalmología durante el curso de la candidemia. ${ }^{(2,14)}$

Los factores de riesgo que se observaron con mayor frecuencia, también se han descrito en mayor o menor medida en estudios previos. $^{(1,3,4,7,10,17,22)}$ El uso de la vancomicina es importante debido a la asociación entre $S$. aureus y Candida sp., en especial tipo albicans, ambos productores de biopelículas y que pueden coexis- tir en dispositivos intravasculares tales como catéteres venosos centrales, marcapasos y cardiodesfribriladores. ${ }^{(20)}$ La presencia del catéter venoso central, es también un factor modificable asociado a la mortalidad en diferentes estudios publicados, en nuestro estudio constituye el segundo factor de riesgo más importante para el desarrollo de un episodio de candidemia. $C$. parasilopsis fue la más frecuentmente aislada, lo cual coincide con lo reportado internacionalmente en los últimos años, en donde se ha visto un incremento en la incidencia de esta especie, asociada entre un 10 y $20 \%$ de los casos, a la presencia de dispositivos intravasculares y al uso de la nutrición parenteral. ${ }^{(19)}$

La presencia de nutrición parenteral, prolongada en la mayoría de los casos, con un promedio no menor de 30 días, fue un factor de riesgo importante, predominando en pacientes quirúrgicos con laparotomía abdominal, en su mayoría por neoplasia del tracto gastrointestinal. La presencia de C. albicans es superior a lo reportado en algunas revisiones internacionales, en donde se reporta hasta un $16 \%$ de episodios de candidemia $^{(11,19)}$, contra un $48 \%$ reportado en el presente trabajo.

Se documentó únicamente ocho pacientes con neoplasia hematológica que desarrollaron un episodio de candidemia, siendo la más frecuente la leucemia mielocìtica aguda (5/8); sin embargo, todos tenían neutropenia absoluta y profilaxis con fluconazol vía oral, debido al uso de quimioterapia antineoplásica, el cual constituye el principal factor de riesgo para sufrir una candidemia. La frecuencia de asilamiento de C.glabrata y $C$. krusei coincide con la literatura internacional, en donde en los últimos años se ha visto un aumento en la incidencia de candidemias asociadas a estos patógenos en este grupo particular de pacientes, condicionado por el uso previo de azoles, además cabe destacar que estas especies en particular tiene resistencia intrínseca a este grupo particular de tratamiento antifúngico ${ }^{(1,2,9)}$.

Por especies, $C$. albicans continúa siendo la más frecuentemente aislada, responsable del 36\% de los episodios de candidemia, al comparar con estudios previos en nuestro nosocomio, el estudio de los Dres. Caballero y Boza en el año 1999, demostró que al igual que ahora, la especie más frecuente era $C$. albicans pero con una prevalencia mayor del 47\%, en el Hospital México, en los últimos cuatro años, según el estudio epidemio- 
lógico realizado por el Dr. Villalobos Vindas, la especie más frecuente es $C$. parasilopsis, seguida por C. albicans; lo anterior a la inversa de lo que ocurre en el Hospital San Juan de Dios.

Así mismo, con el paso de los años se observa un aumento, en la prevalencia mayor de las especies no albicans como tal, siendo las más importantes en orden de frecuencia $C$. parasilopsis y C. tropicalis; lo anterior ya ha sido descrito en otros países. $^{(4,11,12)}$

Otros autores han reportado que C. parasilopsis se asocia más a la presencia del catéter venoso central $^{(1)}$ y en este estudio se demostró dicha asociación, ya que en todos los casos, la permanencia del mismo fue prolongada superior a las 2 semanas.

La estrecha relación mostrada por $C$. tropicalis con el uso de antibióticos, corrobora su considerable potencial biológico como un oportunista ante un huésped comprometido por la supresión de la flora bacteriana normal, como ha sido descrito. $^{(2)}$

Con respecto al tratamiento empleado, debido a que en la mayoría de los casos fue indicado a discreción y criterio clínico de los médicos tratantes y en forma empírica, no se puede establecer los criterios de juicio que se tuvieron para elegir el agente antifúngico empleado, en la mayoría, el tratamiento indicado al inicio fue la anfotericina B, de acuerdo con las guías para el tratamiento de la candidemia publicadas por la Asociación Americana de Infectologìa en el año $2009^{(14)}$, las cuales aún están vigentes. En el tratamiento para los pacientes no neutropénicos, la anfotericina B deoxicolato (AmB-d) administrada a una dosis de 0,5 a $1,0 \mathrm{mg} / \mathrm{kg}$ diarios, como fue administrada en la mayoría de los casos, es una alternativa razonable si existe intolerancia o disponibilidad limitada de otros anti fúngicos (A-I), lo cual sucede en nuestro hospital. Entre los pacientes con candidemia por C. albicans la mayoría (14/22), el tratamiento de inicio fue la anfotericina $\mathrm{B}$, con un promedio de dosis acumulada de $400 \mathrm{mg}$. Se desarrolló insuficiencia renal aguda en el 63\% (9/22), el cual es el efecto secundario más frecuente asociado al uso de este fármaco, lo cual es similar a lo descrito por otros autores. ${ }^{(1,15)}$

En el caso de los pacientes con candidemia debida a $C$. parasilopsis, la mayoría recibió trata- miento empírico inicial con anfotericina $\mathrm{B}$ deoxicolato, lo cual según las guías de candidemia es un tratamiento razonable si existe limitación en cuanto a la disponiblidad de tratamientos antifúngicos (A-I). ${ }^{(14)}$

En el grupo de pacientes neutropénicos incluidos en el presente estudio, que corresponden a aquellos con neoplasia hematológica, tomando en cuenta que las especies más frecuentes correspondieron a $C$. glabrata y $C$. krusei y que en todos los casos tuvieron exposición a profilaxis antifúngica con fluconazol oral y dichas especies poseen resistencia intrínseca al fluconazol, el tratamiento inicial con anfotericina B intravenosa es adecuado y si bien es cierto las guías recomiendan las presentaciones de formulación lipídica preferiblemente, se hace la salvedad de que en aquellos centros como el nuestro, en donde existe disponibilidad limitada de antifúngicos, las presentaciones de la anfotericina B deoxicolato (AmB-d), constituyen una alternativa razonable, ya que los azoles no deben usarse para terapia empírica en pacientes que hayan recibido un azol para profilaxis (B-II). Cabe destacar que en la mitad de los pacientes (4/8), se retiró el catéter venoso central como lo recomiendan las guías. ${ }^{(14)}$

En el presente estudio, se evidenció varias limitantes principales, la primera corresponde a que en el Hospital San Juan de Dios no se cuenta con la realización de pruebas de sensibilidad antifúngica y por ende se desconoce la sensibilidad antifúngica a los tratamientos disponibles de las cepas existentes.

En segundo lugar, las guías de tratamiento de para la candidemia, claramente establecen que una vez diagnosticada, el tratamiento empírico, debe iniciarse dentro de las siguientes 24 horas posteriores a un cultivo de sangre con resultado de levaduras positivo, en el presente estudio, el tratamiento empírico, se inició en el 55,5\% $(34 / 61)$, en promedio a las 72 horas posteriores al resultado del hemocultivo, lo cual se asocia a una mayor mortalidad. ${ }^{(14)}$

En tercer lugar, las guías de tratamiento, establecen claramente que para el tratamiento de la candidemia debe obtenerse cultivos de sangre de seguimiento de todos los pacientes, para garantizar la desaparición del torrente sanguíneo. El panel de expertos recomienda que los cultivos de sangre se realicen a diario, o día por medio, hasta que ya no presenten levaduras. Lo anterior no se 
cumplió en ninguno de los casos incluidos en el presente estudio. ${ }^{(14)}$

\section{CONCLUSIONES}

La candidemia es un diagnóstico frecuente y a considerar en nuestro medio, afectando principalmente a las personas mayores de 65 años y del género masculino.

La mortalidad atribuible a la candidemia en nuestro hospital es alta, la identificación de los grupos susceptibles, los factores de riesgo así como el inicio del tratamiento en forma temprana son determinantes en la evolución clínica y la mortalidad.

La estancia hospitalaria prolongada, asociada al uso de tratamiento antibiótico prolongado y de amplio espectro, la presencia del catéter venoso central, la ventilación mecánica prolongada y la nutrición parenteral, constituyen los principales factores de riesgo para el desarrollo de candidemia en los pacientes hospitalizados.

La especie más frecuente causante de candidemia en nuestro hospital sigue siendo $C$. albicans; $\sin$ embargo existe un incremento en las especies no albicans sobre todo en el grupo de pacientes con neoplasia hematológica.

El tratamiento empírico con anfotericina $\mathrm{B}$, es razonable en nuestro medio, dada la limitación de los tratamientos antifúngicos disponibles y la ausencia de pruebas de sensibilidad antifúngica.

Es necesario implementar la realización de las pruebas de sensibilidad antifúgica en nuestro hospital, con el fin de ofrecer un tratamiento dirigido y oportuno a los pacientes, que permita disminuir la mortalidad atribuible por esta causa. Es necesario implementar la toma de los hemocultivos seriados posteriores al diagnóstico e inicio del tratamiento antifúngico, como lo establecen las guías, con el fin de asegurar la adecuada respuesta y tiempo necesario de tratamiento en este grupo de pacientes.

\section{REFERENCIAS BIBLIOGRÁFICAS}

1. Almirante B Cuenca Estrella M. Candidemia. Enfer Infecc Microbiol Clin 2011;29:325-327.
2. Caballero E Boza R. Candidiasis Sistémica. Experiencia en el hospital San Juan de Dios. Rev Costarricense de Ciencias Médicas 1999;1-10.

3. Osorio JJ Román AR Torre-Cisneros J. Espectro y factores de riesgo de la infección fúngica invasora. Enferm Infecc Microbiol Clin 2007;25:467-476.

4. Conde-Rosa A Amador R Pérez-Torres D et al. Candidemia Distribution, Associated Risk Factors and Attributed Mortality. PR. Health Sci J 2010;29:26-29.

5. Falagas ME Roussos N Vardakas KZ. Relative frequency and the various non-albicans Candida spp among Candidemia isolates from inpatients in various parts of the world. A systematic review. Int $\mathrm{J}$ Infec Dis 2010;14:954-966.

6. Fortin J. Infecciones asociadas a dispositivos intravasculares utilizados para la terapia de infusión. Enferm Infecc Microbiol Clin 2008;26:168-74.

7. Gavaldá J Ruiz I. Guidelines for the treatment of invasive fungal infection. Invasive fungal infection by Candida spp. Invasive Fungal Infection Study Group (MICOMED) and Infection in Transplantation Study Group (GESITRA) of the Spanish Society for Infectious Diseases and Clinical Microbiology (SEIMC). Enferm Infecc Microbiol Clin 2003;21:498-508.

8. Hernández Sierra B Prieto Palomino MA Curiel Balsera E Muñoz Bono J Quesada García G Arias Verdú MD. Perfil Clínico Epidemiológico y taxonómico de la candidiasis sistémica en una unidad de cuidados intensivos. Medicina Intensiva 2009;33:144147.

9. Iñiguez RC Morales CI Rivera ME. Colonización por Candida en enfermos en estado crítico. Acta Médica Grupo Ángeles 2009; 7:75-80.

10. Han SS Yim JJ Yoo CG Kim YW Han SK Shim YS Lee SM. Clinical Characteristics and Risk Factors for Nosocomial Candidemia in Medical Care Units. J Korean Med Sci 2010;25:671-676.

11. León C Ruiz-Santana S Saavedra P et al. Usefulness of the "Candida Score" for discriminating between Candida colonization and invasive candidiasis in non neutropenic critically ill patients: A prospective multicenter study. Crit Care Med 2009;37:16241633. 
12. Leroy O Mir JP Montravers P Gangneux JP Lortholary O AmardCand Study Group. Comparison of albicans vs non albicans Candidemia in French intensive care units. Crit Care Med 2010;14:1-6.

13. Nucci M Queiroz-Telles F Tobón AM Restrepo A Colombo AL. Epidemiology of opportunistic Fungal Infections in Latin America. Clin Infec Dis 2010;51:561-570.

14. Pappas P Kauffman CA Andes D et al. Clinical Practice Guidelines for the Management of Candidiasis: 2009 Update by the Infectious Disease Society of America. Clin Infec Dis 2009;48:503-535.

15. García MS. Agentes antimicóticos para la prevención de infecciones micóticas en pacientes no neutropénicos en estado crítico. Medicina Intensiva 2006;30:354-358.

16. Rodríguez-Hernández M J Ruiz-Pérez de Pipaon M Márquez-Solero $\mathrm{M}$ et al. Candidemias. Enferm Infecc Microbiol Clin 2011;29:328-333.

17. Salavert M. Aspectos cambiantes de la Candidemia. Cont Calid 2007; 1-18.

18. Shorr AF Tabak YP Johannes RS Sun X Spalding J Kollef MH. Candidemia on presentation to the hospital: development and validation a risk score. Critical Care 2009;13:1-10.

19. Stratman RC Martin CA Rappo RP Berger R Magnuson B. Candidemia Incidence in Recipients of Parental Nutrition. Nutrition in Clinical Practice 2010;25:282-289.

20. Uppuluri P Chaturvedi AK Srinivasan A et al. Dispersion as an Important Step in the Candida albicans Biofilm Developmental Cycle. Pathogens 2010;26:1-13.

21. Moudgal V Sobel J. Antifungals to treat Candida albicans. Expert Opin Pharmacother 2010;11:2037-2048.

22. Sampalo Camargo TZ Marra AR Sivla CV et al. Secular trends of Candidemia in tertiary care hospital. Am J Infect Control 2010;38:546-551. 